\title{
Generation of FGF reporter transgenic zebrafish and their utility in chemical screens
}

\author{
Gabriela A Molina1, Simon C Watkins ${ }^{2}$ and Michael Tsang*1,3
}

Address: ${ }^{1}$ Department of Molecular Genetics and Biochemistry, University of Pittsburgh, School of Medicine. BST3-5062, 3051 Fifth Avenue, Pittsburgh, PA 15213, USA, 2Department of Cell Biology and Physiology, Center for Biological Imaging, University of Pittsburgh, School of Medicine S362 Biomedical Science Towers, 3500 Terrace Street. Pittsburgh, PA 15261 USA and ${ }^{3}$ LMG/NICHD/NIH, Building 6B, 9000 Rockville Pike. Bethesda, MD 20892 USA

Email: Gabriela A Molina - gam20@pitt.edu; Simon C Watkins - swatkins@pitt.edu; Michael Tsang* - tsang@mgb.pitt.edu

* Corresponding author

Published: 6 June 2007

BMC Developmental Biology 2007, 7:62 doi:10.1186/147I-2/3X-7-62
Received: 26 January 2007

Accepted: 6 June 2007

This article is available from: http://www.biomedcentral.com/I47I-2I3X/7/62

(C) 2007 Molina et al; licensee BioMed Central Ltd.

This is an Open Access article distributed under the terms of the Creative Commons Attribution License (http://creativecommons.org/licenses/by/2.0), which permits unrestricted use, distribution, and reproduction in any medium, provided the original work is properly cited.

\begin{abstract}
Background: Fibroblast Growth Factors (FGFs) represent a large family of secreted proteins that are required for proper development and physiological processes. Mutations in mouse and zebrafish FGFs result in abnormal embryogenesis and lethality. A key to understanding the precise role for these factors is to determine their spatial and temporal activity during embryogenesis.
\end{abstract}

Results: Expression of Dual Specificity Phosphatase 6 (dusp6, also known as Mkp3) is controlled by FGF signalling throughout development. The Dusp6 promoter was isolated from zebrafish and used to drive expression of destabilized green fluorescent protein (d2EGFP) in transgenic embryos $(\mathrm{Tg}$ (Dusp6:d2EGFP)). Expression of d2EGFP is initiated as early as 4 hours post-fertilization (hpf) within the future dorsal region of the embryo, where $f g 3$ and fgfo are initially expressed. At later stages, d2EGFP is detected within structures that correlate with the expression of Fgf ligands and their receptors. This includes the mid-hindbrain boundary (MHB), pharyngeal endoderm, otic vesicle, hindbrain, and Kupffer's vesicle. The expression of d2EGFP is under the control of FGF signalling as treatment with FGF Receptor (FGFR) inhibitors results in the suppression of d2EGFP expression. In a pilot screen of commercially available small molecules we have evaluated the effectiveness of the transgenic lines to identify specific FGF inhibitors within the class of indolinones. These compounds were counter screened with the transgenic line $\operatorname{Tg}(F l l: E G F P) y l$, that serves as an indirect read-out for Vascular Endothelial Growth Factor (VEGF) signalling in order to determine the specificity between related receptor tyrosine kinases (RTKs). From these assays it is possible to determine the specificity of these indolinones towards specific RTK signalling pathways. This has enabled the identification of compounds that can block specifically the VEGFR or the FGFR signalling pathway.

Conclusion: The generation of transgenic reporter zebrafish lines has allowed direct visualization of FGF signalling within the developing embryo. These FGF reporter transgenic lines provide a tool to screen for specific compounds that can distinguish between two conserved members of the RTK family. 


\section{Background}

The complex process of embryogenesis is directed by the regulation of signalling pathways that are achieved in part by the activity of a variety of secreted ligands. Thus understanding the temporal and spatial activity of signalling peptides is key to determining the role for these factors in controlling cellular fates. For example, Fibroblast Growth Factors (FGFs), a family of secreted glycoproteins, perform crucial functions that include the establishment of embryo polarity, the formation of organizing centres, and the induction of limb outgrowth [1-3]. These ligands are expressed in discrete domains during development and their actions are restricted to cells that express integral membrane proteins that can bind FGFs [2,4]. The FGF receptors (FGFRs) are members of the receptor tyrosine kinase (RTK) class of transmembrane proteins and activate several signalling cascades, including the phospholipase C gamma (PLC- $\gamma$ ), phosphatidylinositol-3 kinase (PI3K) which activates Akt/protein kinase B, and Ras which activates extracellular signal-regulated protein kinase (ERK, also known as MAPK) pathways [5,6]. FGF activity results in the control of gene expression through the modification of transcription factors by activated ERKs and AKT. As a consequence of altered gene expression, cellular proliferation, survival and fate determination can be governed by FGF activity. How FGFs control gene expression and the nature of the genes that they regulate during development is still not completely established.

One step towards defining FGF target genes is to determine the temporal and spatial activity of FGFs during development. This will provide an activity map of where and when these factors act to control developmental processes. Since activation of FGF signalling results in the phosphorylation of Erk, one approach to illustrate FGF activity during development has been to detect the spatial and temporal presence of phosphorylated ERKs in the embryo. This has resulted in mapping the location of FGF activity during mouse, chick, Xenopus laevis and zebrafish embryogenesis [7-12]. While these studies provide a detailed analysis of FGF activity during development, it is not possible to visualize FGF activity in the live embryo and observe the dynamic changes in FGF signalling as the embryo develops.

We have previously identified several FGF regulated genes in zebrafish, including the Dual Specificity Phosphatase 6, dusp6 (also known as Map Kinase Phosphatase 3, mkp3), and Sef, two genes that exhibit almost identical expression to $f g f 8$ and $f g f 3$ during development $[11,13-15]$. Dusp 6 functions to dephosphorylate activated p44 and p42 ERKs and over-expression of Dusp6 results in the suppression of FGF activity in the embryo [11,16-18]. Expression of dusp6 was suppressed in embryos treated with SU5402, an FGFR inhibitor, or by the ectopic expression of dominant nega- tive FGFR, indicating that $d u s p 6$ transcription is regulated by FGFs [11,16-18]. Genetic studies in mouse have identified the requirement for FGFRs in maintaining Dusp6 expression, as loss of either FGFR1 or FGFR2 resulted in the depletion of Dusp6 transcripts [19]. It is clear from these studies that Dusp6 expression is regulated by FGF ligands and receptors, however it has been controversial as to which signalling pathway downstream of the receptor is required for Dusp6 gene transcription.

Experiments described in the chick, mouse and zebrafish embryos have provided clues that Dusp6 gene regulation is context dependent $[11,16-18,20,21]$. In several studies, the PI3K inhibitor, LY294002, was used in the chick limb bud to show that blocking the PI3K/AKT pathway results in the suppression of Dusp6 expression within the distal limb bud $[17,20]$. Likewise, implantation beads soaked in LY294002 could suppress Dusp6 expression within the mid-hindbrain boundary (MHB) in mouse and chick embryos [18,21]. In contrast, genetic studies in the mouse has revealed that the $\mathrm{PI} 3 \mathrm{~K}$ pathway is not required as knock-out of PDK1, an upstream activator of PI3K, still allowed expression of Dusp6 albeit in a disorganized fashion [20].

Analysis of the RAS/MAPK pathway under similar experimental conditions indicates that this pathway is also important for Dusp6 gene expression. For instance, the implantation of beads soaked with PD184352, a specific inhibitor of Mek can also block Dusp6 expression in the chick limb bud and somites, implicating the RAS/MAPK pathway in regulating Dusp6 expression [16,20,22]. Further, over-expression of Dusp6 itself, which would result in the removal of activated ERKs and hence the shutdown the RAS/MAPK pathway, resulted in the down regulation of Dusp6 transcription $[11,20]$. Although these studies highlight the complex nature of Dusp6 gene regulation downstream of the FGF receptor it is however clear that Dusp6 is a direct target of FGF signalling. This concept is supported by the fact that the presence of Erk phosphorylation correlates with Dusp6 expression throughout chick embryogenesis [12]. A detailed map of ERK activation was described and Dusp6 expression was found to colocalize with activated ERKs in all tissues throughout chick development [12]. Thus FGF activity can be directly measured by either the presence of phosphorylated ERKs or indirectly by the presence of Duspo transcripts.

The generation of transgenic lines that express fluorescent reporters in response to FGFs would allow live visualization of FGF activity during development. Since zebrafish embryos develop ex utero, the direct visualization of d2EGFP in the embryos can provide an indirect biosensor for FGF activity in vivo. In this report we have generated transgenic zebrafish lines that expresses a destabilized 
form of Green Fluorescent Protein (d2EGFP) under the control of active FGF signalling. To achieve this we isolated the promoter region of the $d u s p 6$ gene and fused it to d2EGFP reporter gene. d2EGFP fluorescence can be visualised in transgenic embryos within multiple tissues where activated ERKs (phosphorylated) have been detected, and where expression of FGF ligands, receptors and target genes such as sef, sprouty4, pea3 and erm have been described $[9,10,13,15,23-31]$.

The zebrafish has become a viable model organism for chemical screens [32-34]. With the generation of fluorescent transgenic reporter zebrafish lines it is possible to discover specific molecules that can alter differentiation events during organogenesis [35]. We have validated the FGF reporter lines as tools to identify novel FGF modulators and in counter screens it is possible to determine compound specificity towards two closely related RTK pathways. The generation of in vivo reporters for FGF activity will provide a valuable tool to screen for genes or chemicals that modulate FGF signalling.

\section{Results}

\section{Generation of transgenic FGF reporter lines}

The zebrafish Dusp6 gene locus was identified by PCR screening a BAC library from Genome Systems. The BAC clone contained the full gene Dusp6 locus, and a $10 \mathrm{~Kb}$ fragment that includes the $5^{\prime}$ untranslated sequence within exon I was subcloned into a vector containing the $d 2 E G F P$, a gene that encodes a destabilised green fluorescent protein that has a two hour half-life (Figure 1A) [36]. Since FGF signalling during embryogenesis is dynamic, we reasoned that a destabilized form of EGFP would likely recapitulate the endogenous expression of dusp6 mRNA. 1-cell stage zebrafish embryos were co-injected with the transgenic DNA construct and I-Sce 1 meganuclease as described by Thermes et al. to generate transgenic lines [37]. Injected embryos were raised to adulthood and screened for potential germline carriers by d2EGFP expression. Ten founder transgenic animals were identified in the F1 generation. While d2EGFP expression levels differed between each of the transgenic lines, the expression patterns were identical between the transgenic lines. We maintained four of the strongest lines (Tg(Dusp6:d2EGFP) $)^{p t 6-p t 9}$ ), and for this study we utilized the line $T g$ (Dusp6:d2EGFP) ${ }^{p t 6}$. We have generated homozygous lines for several of the transgenes and observed the expected Mendelian inheritance. Further, there were no deleterious effects noted in transgenic animals, suggesting that insertion of the DNA construct did not impact zebrafish growth and development.

\section{Expression of d2EGFP is localized to FGF signalling centres in transgenic embryos}

d2EGFP was first visualized as early as dome stage within the prospective dorsal region of the embryo, consistent
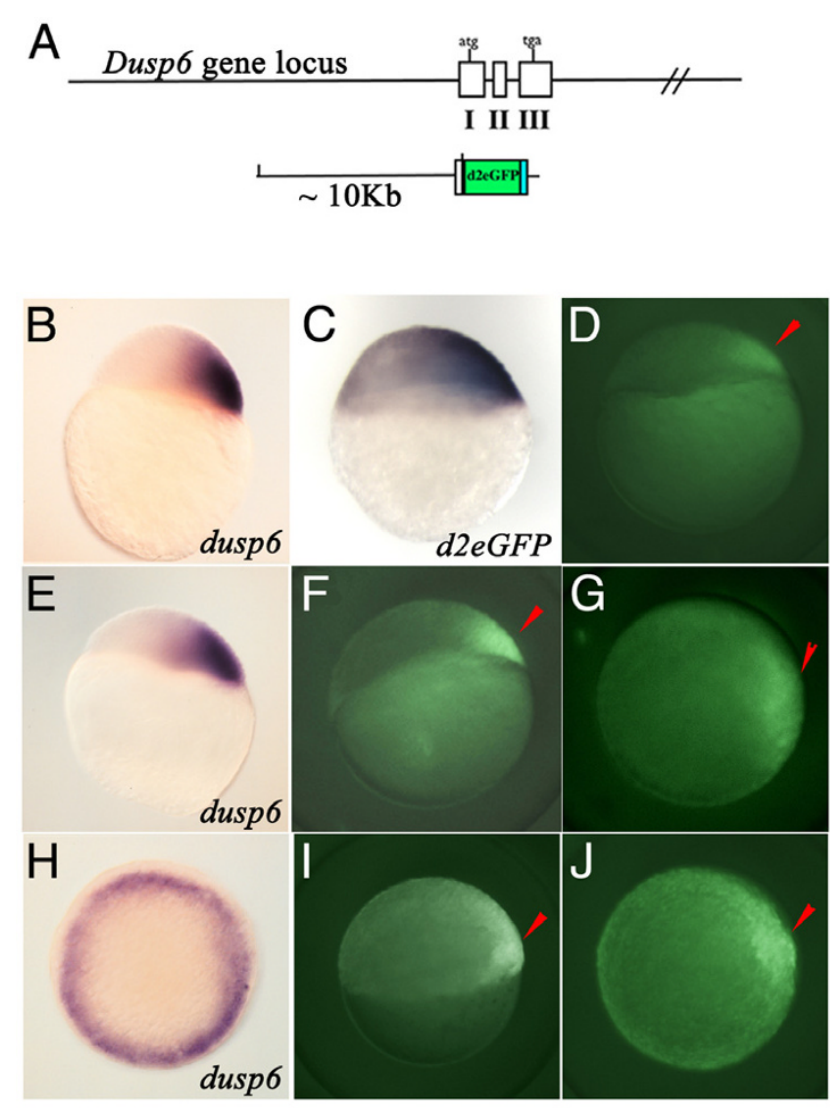

\section{Figure I}

Generation of Dusp6 DNA construct and expression of d2EGFP in transgenic embryos. (A) Diagram showing the Dusp6 gene locus and the DNA construct used in generating transgenic zebrafish. (B, E, \& $\mathbf{H})$ dusp6 expression at oblong (B), dome (E), and shield stage. (C) d2EGFP mRNA expression at sphere stage. (D, F, G, I, \& J)

$\mathrm{Tg}$ (Dusp6:d2EGFP)pt6 embryos at dome (D), 30\% epiboly (F \& G), and shield (I \& J) stage. (B-F \& I) are lateral views and (H \& J) are animal views. Red arrowheads mark dorsal region of the embryo.

with the restricted dorsal expression of dusp $6 m R N A$ and with the initial expression patterns of $f g f 3$ and -8 (compare Figure $1 \mathrm{~B}$ to $1 \mathrm{D}$ ) [11]. The fluorescent pattern was supported by the expression of $d 2 E G F P ~ m R N A$ as tested by in situ hybridisation (Figure 1C). At sphere stage, d2EGFP expression is maintained within the dorsal region and colocalises with dusp6 expression, a domain where the organiser (shield) eventually forms (compare Figure 1E to $1 \mathrm{~F})$. At $6 \mathrm{hpf}$, the highest d2EGFP expression is detected within the shield, and weakly throughout the margin, which resembles the pattern of dusp6 mRNA at this stage (compare Figure $1 \mathrm{~J}$ to $1 \mathrm{H}$ ).

Reporter gene expression was also analysed at later stages by in situ hybridisation performed to detect the presence of d2EGFP $m R N A$. At bud stage (10 hpf), d2EGFP tran- 
scripts can be detected within the posterior ventral domain and within the presumptive hindbrain, where $f g f 8$ expression has been noted (Figure 2A) [14,30,31]. By $24 \mathrm{hpf}$, d2EGFP $m R N A$ is detected within the MHB, pharyngeal arches, otic vesicle, retina, optic stalk and dorsal diencephalon (Figure 2B). The ligands $f g f 8, f g f 17$ and $f g f 3$, and FGFRs are known to be expressed within these same domains, suggesting that $m R N A$ expression of this reporter gene is under the control of FGF signalling $[14,23,25,26,30,31]$. In comparison to the fluorescent protein expression, $22 E G F P$ transcripts can be detected in a much wider domain and also more prominent (compare Figure $2 \mathrm{~A}$ to $2 \mathrm{C}$ and Figure $2 \mathrm{~B}$ to $2 \mathrm{I}$ ). One reason for the discrepancy can be attributed to the threshold level
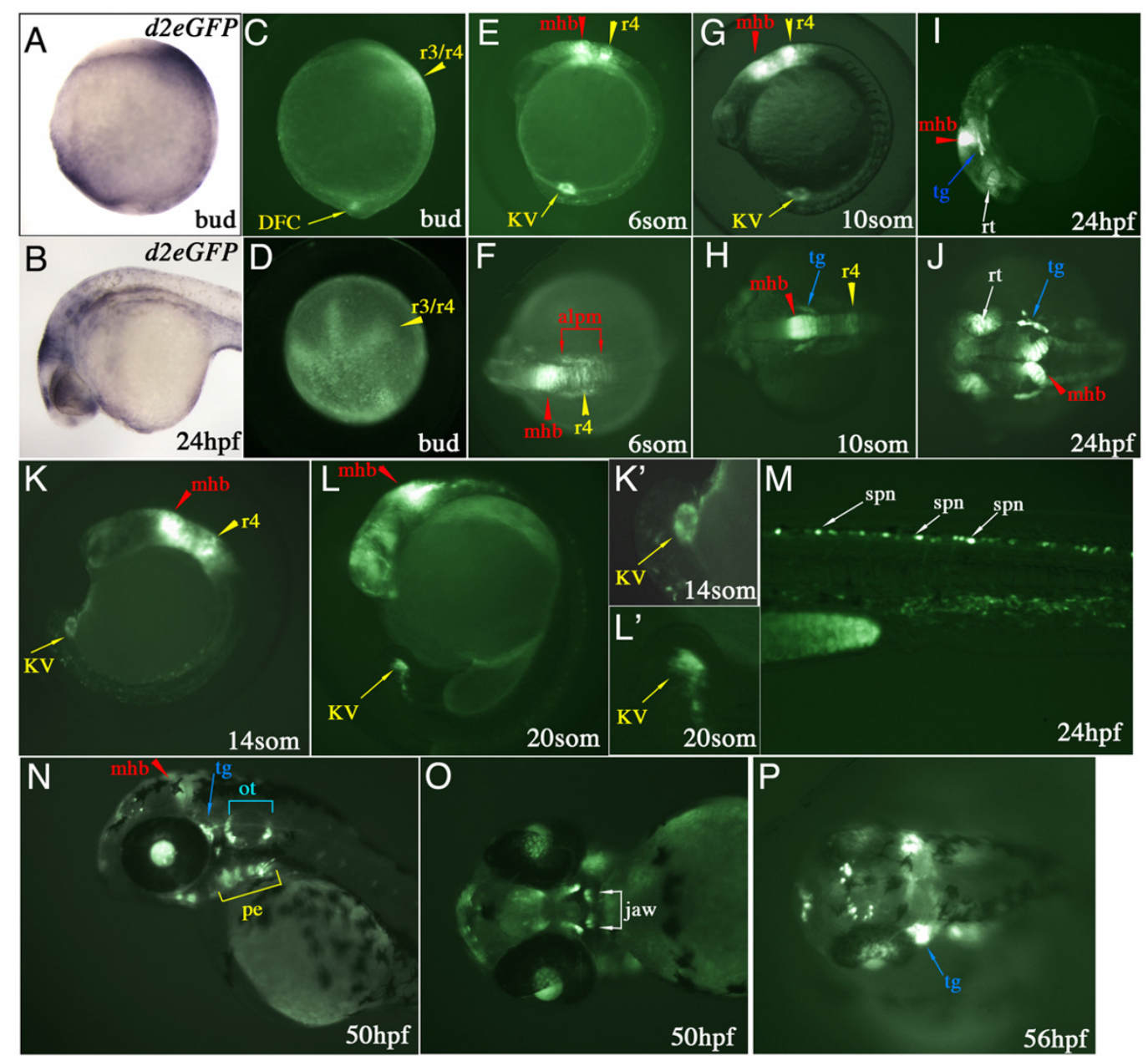

\section{Figure 2}

Spatial and temporal d2EGFP expression in Tg(Dusp6:d2EGFP)pt6 embryos. (A \& B) Lateral views of $d 2 E G F P$ mRNA expression at Bud stage and 24 hpf. (C-P) d2EGFP expression in $\mathrm{Tg}($ Dusp6:d2EGFP)pt6 embryos, stages are indicated in each panel. At bud stage (C \& D), d2EGFP is detected in the hindbrain ( $r 3 / r 4$, yellow arrowhead) and within the caudal region in the DFCs. (E, G \& K) From 8- to I4-somite stages, lateral views show expression of d2EGFP in cells lining Kupffer's vesicle, within r4 (r4, yellow arrowhead) and the mid-hindbrain boundary (mhb, red arrowhead). (F \& $\mathbf{H})$ Dorsal views show high d2EGFP expression within the MHB, r4 and the anterior lateral plate mesoderm (alpm, red brackets). (H) At I0-somite stage initial d2EGFP expression is detected within the trigeminal ganglia (tg, blue arrow). (I \& J) 24 hpf embryo showing d2EGFP expression in the MHB, trigeminal ganglia, dorsal retina ( $r$, white arrow) and pharyngeal endoderm (pe, yellow bracket). (K \& L) 14 and 20-somite stage embryo highlighting the expression of d2EGFP in Kupffer's vesicle. Higher magnifications are show in (K' \& L'). (M) Trunk region shows d2EGFP expression within the dorsal spinal cord neurons (spn, white arrow) at 24 hpf. (N) At $50 \mathrm{hpf}$ expression is noted in the MHB, trigeminal ganglia, pharyngeal endoderm and otic vesicle (ot, blue bracket). (O) Ventral view of $50 \mathrm{hpf}$, showing d2EGFP expression in the jaw (white bracket). (P) At 56 hpf, strong expression in noted in the trigeminal ganglia, the jaw and also in neurons within the dorsal diencephalon. 
required for visualisation of d2EGFP protein as compared to the detection of transcripts by in situ hybridisation.

At bud stage, fluorescent protein can be visualized within the medial neural plate, presumptive hindbrain and at a position that corresponds to the site where the Dorsal Forerunner Cells (DFCs) in the posterior domain of transgenic embryos have been described (Figure 2C \&2D) $[38,39]$. The DFCs represent a specialized group of noninvoluting cells that migrate just ahead of the shield during gastrulation and eventually forms a fluid filled structure known as Kupffer's vesicle, a transient organ particular to teleosts $[38,39]$. Kupffer's vesicle is thought to serve equivalent functions in establishing left-right polarity as the mouse node [40-43].

By the 6-somite stage, strong d2EGFP fluorescence can be detected in the MHB, the hindbrain with strongest expression in $\mathrm{r} 4$, the anterior lateral plate mesoderm and the Kupffer's vesicle (Figure 2E \&2F). Approximately two hours later at the 10-somite stage expression of d2EGFP is located within the same domains as described for the 6somite stage with the addition of the trigeminal ganglia (Figure 2G \&2H). The expression within Kupffer's vesicle is quite striking as the structure is completely outlined by d2EGFP positive cells from the 6-somite onwards (Figure $2 \mathrm{C}, 2 \mathrm{E}, 2 \mathrm{G}, 2 \mathrm{~K}$ and $2 \mathrm{~K}$ ). In the zebrafish, a role for FGF signalling has been suggested in the formation of Kupffer's vesicle, as $f g f 8$ is expressed in the DFCs at gastrulation, and this structure is absent in about $30 \%$ of ace(fgf8) mutants [44]. The Tg(Dusp6:d2EGFP) ${ }^{p t 6}$ line confirms that the DFCs and Kupffer's vesicle receive FGF signals from the time when the DFCs begin to coalesce and right through to the 20-somite stage when Kupffer's vesicle begins to collapse (Figure 2K, 2L, 2K', \&2L'). Time lapse imaging of the transgenic embryos shows fluorescent DFCs migration towards the posterior region of the embryo and the formation of Kupffer's Vesicle at the 6somite stage [see Additional files 1, 2, 3 and 4]. d2EGFP expression is still noted throughout these stages and even after the collapse of Kupffer's vescles when these epithelial cells migrate towards the tail bud and contribute to mesodermal tissues such as notochord, posterior somites and the tail bud (see Additional files 2, 3 and 4) [38,39]. By the 26-somite stage, the Kupffer's vesicle cells have lost their expression of d2EGFP, suggesting that these cells no longer receive FGF signals (data not shown).

At $24 \mathrm{hpf}$, d2EGFP can be visualized within the dorsal retina, trigeminal ganglia, otic vesicles, within the dorsal diencephalon, and dorsal spinal cord neurons (Figure 2I, 2J \&2M). These domains of d2EGFP expression are consistent with expression of FGF ligands, receptors and known target genes such as erm, pea3, sef and sprouty4, suggesting that the transgenic line reports on FGF activity in vivo [13,15,24,27-29]. d2EGFP expression persists throughout the next day of development in a majority of the same domains as at $24 \mathrm{hpf}$ and continues up to $56 \mathrm{hpf}$ (Figure 2N, 2O \&2P). The one exception is that dorsal retina expression is lost, while lens expression becomes stronger from 36 hpf onwards (Figure $2 \mathrm{~N}$ ). Expression at later stages can also be detected within the developing jaw and further refined within the pharyngeal arches and the pectoral fins (Figure $2 \mathrm{~N} \& 2 \mathrm{O}$, pectoral fins not shown). A dorsal view of a $56 \mathrm{hpf}$ transgenic embryo reveals d2EGFP expression within distinct cells in the dorsal diencephalon, suggesting that these particular cells are responding to FGF signals (Figure 2P). Of particular interest is that there seems to be an asymmetric distribution of fluorescent cells with respect to the left-right axis, implying that within this region of the diencephalon, FGF signalling is asymmetrical (Figure 2P). An alternative view is that the asymmetric expression of d2EGFP is a result of anatomical or stochastic (random) differences between the left and right side of the diencephalon. Studies have shown that the left habenular nuclei is larger and the parapineal gland is situated to the left of the midline, suggesting that d2EGFP expression reflects structural difference between the left and right dorsal diencephalon $[45,46]$.

The domains of d2EGFP expression in the transgenic embryos were consistent with many of the regions where endogenous dusp 6 transcripts have been detected. However, dusp 6 mRNA expression was not fully recapitulated in the transgenic embryos, in particular FGF activity and dusp6 expression have been well documented within the somites and tail bud and yet no expression of d2EGFP $m R N A$ or protein were detected in these regions (Figure 2) [11]. One explanation is that only $10 \mathrm{~Kb}$ of upstream Dusp6 promoter sequence was used to generate the DNA construct for transgenesis, hence it is likely that somitic and tail bud enhancers were not present within this sequence.

In summary we have generated several transgenic lines that express d2EGFP under the control of the Dusp6 promoter, a gene that is directly regulated by FGF signalling during development. Since d2EGFP has a half life of just two hours, it is likely that domains of high d2EGFP expression represents cells that respond to active FGF signalling as opposed to cells inheriting fluorescent protein from their ancestors.

\section{FGF signalling controls reporter gene expression}

To test whether d2EGFP expression is responsive to experimental modulation in FGF signalling, we injected $f g f 8$ mRNA (10 pg) into 2-4 cell stage $T g$ (Dusp6:d2EGFP)pt6 embryos. As expected, expression of d2EGFP was greatly expanded in $f g f 8$-injected embryos at the gastrula stage (compare Figure 3B to 3A). Conversely, injection of $f g f 8$ - 

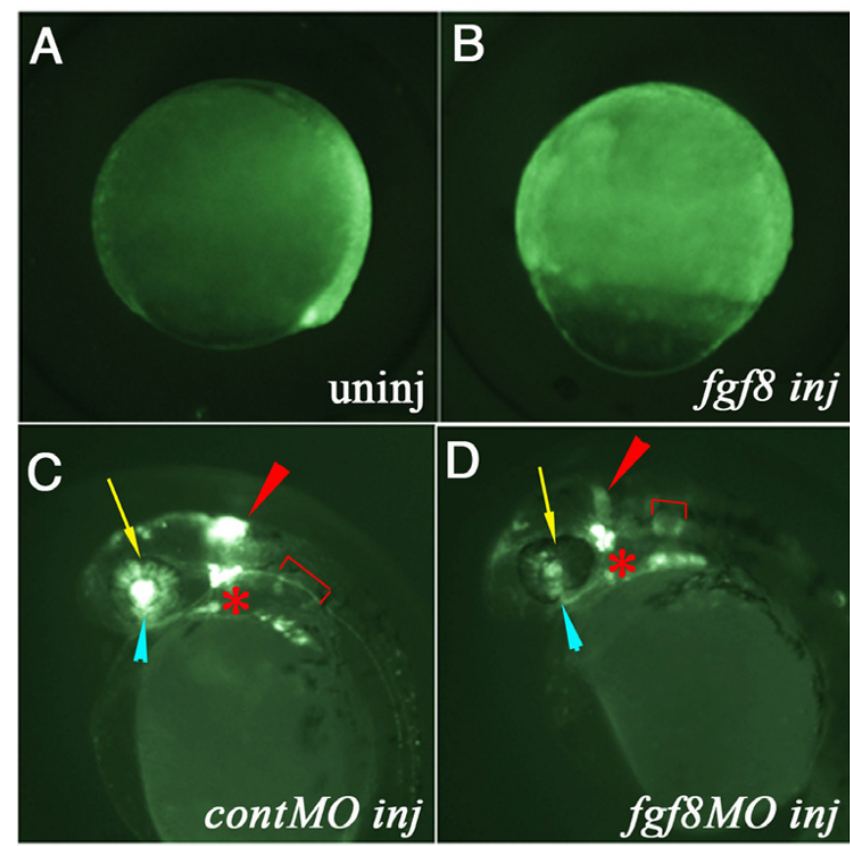

Figure 3

D2EGFP expression is dependant on FGF signaling. (A \& B) Lateral views of gastrula staged $T g($ Dusp6:d2EGFP)pt6 embryos. Control uninjected is shown in (A), while in (B) fgfo $m R N A$ injected at the 2-cell stage. Expression of d2EGFP is greatly expanded in fof8-injected embryos. (C \& D) Lateral views of $28 \mathrm{hpf}$ transgenic embryos. (C) Control MO injected (D) fgf8-MO injected embryo shows loss of d2EGFP expression within the $\mathrm{MHB}$, dorsal retina and smaller otic vesicles. Red arrowhead: MHB; yellow arrow: dorsal retinal; blue arrowhead: optic stalk; red bracket: otic vesicle.

MOs (10 ng) resulted in a suppression of d2EGFP expression within the MHB and retina, while d2EGFP expression within the trigeminal ganglia was unaffected (compare Figure $3 \mathrm{D}$ to $3 \mathrm{C}$ ). To confirm these findings we analyzed d2EGFP expression in two mutant zebrafish lines that exhibit defective FGF signalling $[31,47]$. We in-crossed $T g$ (Dusp6:d2EGFP)pt6 into the ace $(f g f 8)$ mutant and expression of d2EGFP within the MHB was greatly diminished in mutant embryos from 20 hpf onwards (not shown). This is clearly evident at $28 \mathrm{hpf}$ when the MHB is morphologically distinguishable between wildtype siblings and mutants (Figure 4A-D). Furthermore expression within the retina and lens was absent, while expression in the optic stalk and trigeminal ganglia were unaffected at 28 hpf (Figure 4A-D). In the noi (pax2.1) mutant, d2EGFP expression was markedly reduced in the MHB, where FGF signalling is absent, however expression within the retina, lens, trigeminal ganglia and otic vesicles was unaltered. In contrast to the ace mutants, expression of $\mathrm{d} 2 \mathrm{EGFP}$ within the optic stalk is severely reduced as this structure fails to from in the noi mutants (compare Figure 4E to 4F) Taken
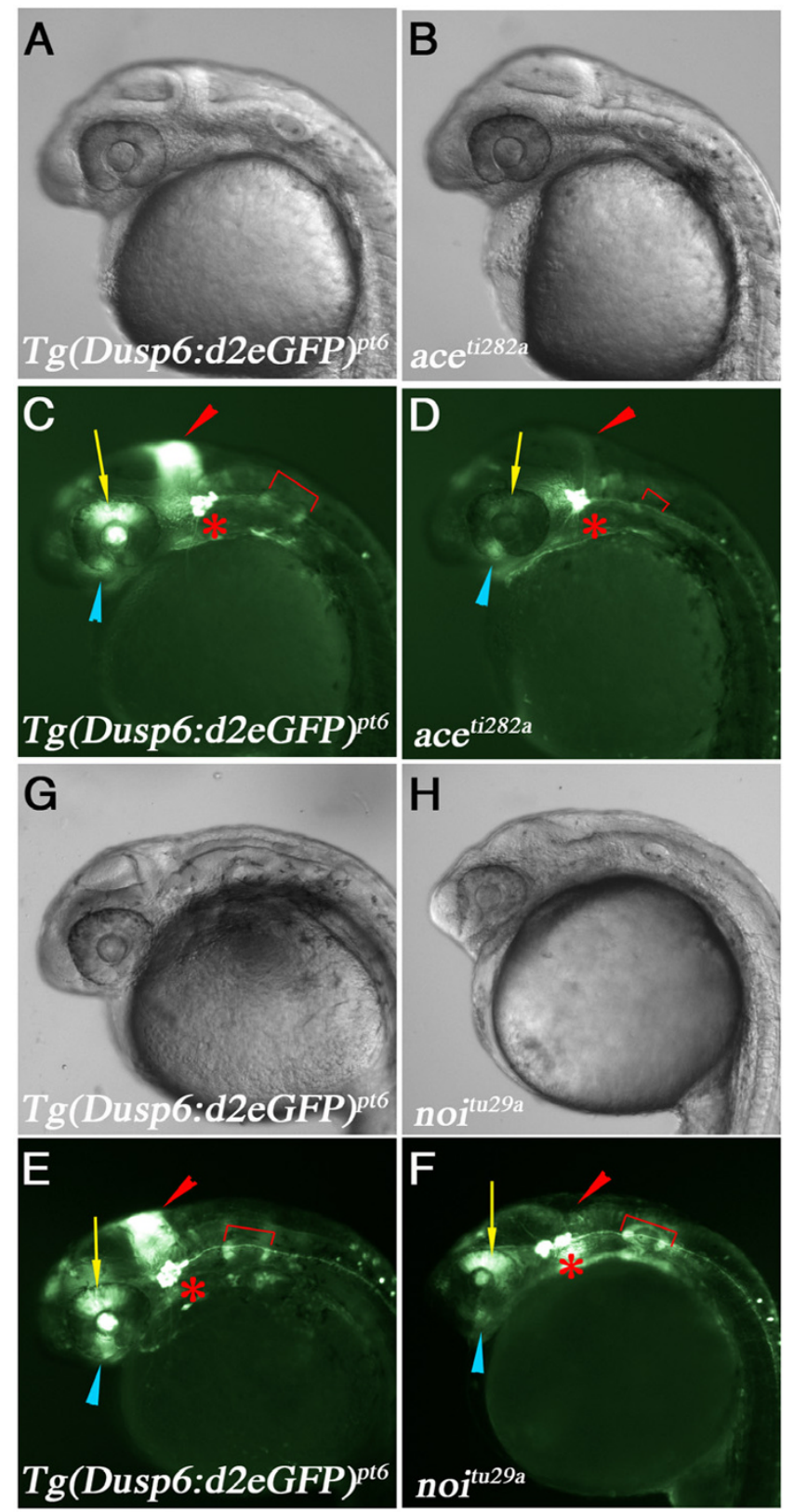

\section{Figure 4}

\section{D2EGFP expression in fgf8/ace and pax2. I/noi}

mutants. (A-F) Lateral views of $T g($ dusp6:d2EGFP)pt6

embryos at $28 \mathrm{hpf}$ crossed into ace or noi mutants. Genotype is listed in bottom left corner. (A \& B) Brightfield images of wildtype sibling and ace mutant embryo, respectively. (C \& D) d2EGFP expression in WT sibling and ace mutant. Note loss of d2EGFP expression within the MHB, dorsal retina, and the smaller otic vesicle, while d2EGFP expression in the trigeminal ganglia is unaffected. ( $(\mathbf{\&} \mathbf{H})$ Brightfield images of wildtype sibling and noi mutant embryo. (E \& F) d2EGFP expression is lost in the MHB and optic stalk. In contrast to the ace mutants, expression within the dorsal retina and otic vesicles are normal. 


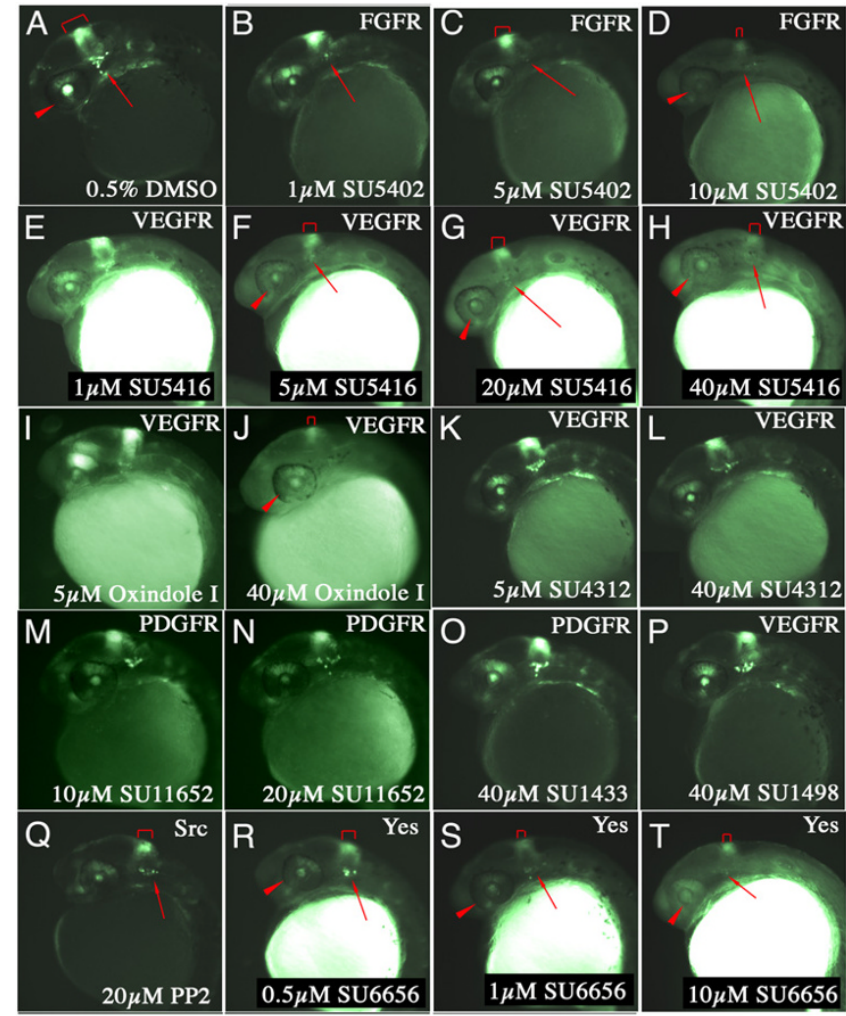

Figure 5

Validation of $\operatorname{Tg}$ (Dusp6:d2EGFP) ${ }^{\text {pt6 }}$ line for chemical screening. (A-Y) Lateral views of $30 \mathrm{hpf}$ embryos treated with the compound and dose are indicated on the bottom right. The protein targets for these compounds are listed in the top right hand corner. (A) Embryo incubated in 0.5\% DMSO as control. (B-D) Increasing doses of SU5402 suppressed d2EGFP expression in the MHB (red bracket), trigeminal ganglia (red arrow) and dorsal retina (red arrowhead). (E-H) Increasing doses of SU54 16, a non-specific inhibitor of VEGFRs, suppressed FGF signalling. (I \& J) Oxindole I another related VEGFR inhibitor also suppressed d2EGFP fluorescence in transgenic embryos. (K-N) In contrast, two compounds with similar chemical structure SU43 I 2 and SUI I652, did not block FGF signalling. (O \& P) Likewise, two unrelated inhibitors of PDGFR and VEGFR, SUI433 and SUI498 also failed to suppress d2EGFP expression. (Q-T) PP2 and SU6656, two Src Kinase inhibitors suppressed FGF signalling in transgenic embryos.

together, these results show that we have generated a transgenic reporter line that expresses d2EGFP under the control of FGF signalling during development.

\section{Treatment of Tg(Dusp6:d2EGFP)pt6 embryos with known FGF pathway inhibitors}

Given that $\operatorname{Tg}$ (Dusp6:d2EGFP)pt6 embryos respond to changes in FGF signalling, these embryos can provide a valuable tool to screen for molecules that modulate FGF signalling in vivo. To validate that these reporter lines can measure changes in FGF activity in a chemical screen, $T g$ (Dusp6:d2EGFP)pt6 embryos were treated with known inhibitors of the FGF pathway. For our studies we selected transgenic embryos at $24 \mathrm{hpf}$ and treated with compounds for 8 hours. We reasoned that since the half-life of d2EGFP is 2 hours, then an inhibitor of FGF signalling should substantially reduce d2EGFP fluorescence after 68 hours of treatment. Furthermore, since embryos at this developmental stage ( $24 \mathrm{hpf}$ ) have already formed many of the structures that express d2EGFP such as the MHB, trigeminal ganglia and otic vesicles, then the assay would directly measure loss of d2EGFP fluorescence as a consequence of FGF inhibition and not per se a loss of these tissues.

We treated transgenic embryos with the indolinone FGF receptor inhibitor, SU5402 [Additional file 5 shows the structure of the compounds used in this study] [48]. SU5402 has been extensively used to block FGF signalling in zebrafish and Xenopus laevis embryos with doses ranging from $20 \mu \mathrm{M}$ to $160 \mu \mathrm{M}[10,27,49,50]$. In this study, 1 $\mu \mathrm{M}$ SU5402 was effective at suppressing expression of $\mathrm{d} 2 \mathrm{EGFP}$ within the trigeminal ganglia and reducing $\mathrm{MHB}$ fluorescence after only 8 hours of treatment (compare Figure $5 \mathrm{~A}$ to $5 \mathrm{~B}$ ). At $5 \mu \mathrm{M}$ of SU5402, expression of d2EGFP was markedly reduced within the $\mathrm{MHB}$, and even eliminated within the dorsal retina and trigeminal ganglia (Figure 5C). At $10 \mu \mathrm{M}$, d2EGFP fluorescence in the transgenic embryos was completely abolished (Figure 5D).

Treatment with other receptor tyrosine kinase inhibitors was also performed to determine the specificity of this assay for identifying other small molecules that can modulate FGF signalling. We assessed the activity of several Vascular Endothelial Growth Factor Receptor (VEGFR) and Platelet Derived Growth Factor Receptor (PDGFR) inhibitors to reveal if these compounds, structurally related to SU5402, could suppress d2EGFP expression in the FGF reporter lines. We reasoned that most of these compounds contain the same indolinone backbone as SU5402, and therefore some would also block FGFR signalling in the zebrafish. Thus the analysis of these compounds in the FGF reporter line can determine the chemical structures that suppress FGF signalling from a family of related molecules. One of the first indolinones to undergo clinical trials was SU5416, a reported specific inhibitor for VEGFR2 [see Additional file 5] [51-53]. SU5416 was added to $24 \mathrm{hpf}$ stage transgenic embryos for 6 hours to determine if this compound is specific for VEGFR versus FGFR. While this compound exhibits autofluorescence, especially within the yolk, the expression of $\mathrm{d} 2 \mathrm{EGFP}$ did not markedly change at $1 \mu \mathrm{M}$ (Figure $5 \mathrm{E})$. In contrast at $5 \mu \mathrm{M}$, the trigeminal ganglia and $\mathrm{MHB}$ fluorescence was markedly diminished (Figure 5F). At even higher doses, SU5416 completely eliminated 
d2EGFP expression within the transgenic embryos, even though the treated embryos exhibited strong fluorescent yolks, the expression of d2EGFP within the MHB was clearly eliminated (Figure 5G \&5H). These results suggest that SU5416 is not completely specific to VEGFR2 at these concentrations and it can block FGF signalling. Similarly, Oxindole I was also effective at blocking d2EGFP expression, however a much higher dose $(40 \mu \mathrm{M})$ was required [see Additional file 5], and (Figure 5I \&5J). SU4312 represents another indolinone inhibitor of VEGFR, which has been shown to block autophosphorylation of VEGFRs [54]. In contrast to SU5416, treatment up to $40 \mu \mathrm{M}$ of SU4312 had little effect in the expression of d2EGFP within the MHB and trigeminal ganglia in the $\mathrm{Tg}$ (Dusp6:d2EGFP) ${ }^{p t 6}$ embryos [see Additional file 5] and (Figure 5K \&5L). A newer generation of indolinone compound was also tested to determine its specificity towards VEGFR/PDGFR versus FGFR. SU11652, has been shown to have at least 100 -fold greater inhibitory effect targeting a VEGFR and PDGFR versus FGFR [see Additional file 5] $[55,56]$. In our assays, SU11652 did not alter d2EGFP expression suggesting that this compound does not block FGF signalling (Figure $5 \mathrm{M} \& 5 \mathrm{~N}$ ). We also treated transgenic embryos with two structurally unrelated VEGF signalling inhibitors, SU1498 and SU1433 (also known as AG1433) to determine the specificity of these molecules [see Additional file 5] [57-59]. Both compounds have been shown to be effective at blocking VEGFR, and to a lesser extent basic FGF in HUVEC tubulogenesis assays [58]. Treatment with high doses of SU1433 or SU1498 did not alter d2EGFP fluorescence in the transgenic embryos, suggesting that these chemically divergent compounds do not block FGF signalling in the zebrafish at the doses indicated (Figure $5 \mathrm{O} \& 5 \mathrm{P}$ ).

We next determined the activity of Src Kinase inhibitors and their role in FGF signalling in the early embryo as previous reports have shown that several Src family members function to relay FGF signalling $[60,61]$. PP2, an inhibitor of several Src Kinases including Fyn and Lck as well as PDGFRs and Bcr-Abl, only mildly altered d2EGFP expression at $20 \mu \mathrm{M}$ (Figure 5Q), a dose that is several fold higher than what has been shown to inhibit Fyn and Lck in cell culture $[62,63]$. SU6656, a more specific inhibitor of Src kinases, exhibited robust inhibitory activity in these assays. At concentrations of $1 \mu \mathrm{M}$, expression of d2EGFP was completely abolished within the MHB, trigeminal ganglia and the retina. The concentration of SU6656 was within the range that is known to be specific for blocking mouse Src kinase activity and not other related tyrosine kinases (Figure 5R-T) [63]. In fact $1 \mu \mathrm{M}$ SU6656 is likely to inhibit only the Src members, Yes, Fyn, Src and Lyn, with Yes kinase exhibiting higher sensitivity, suggesting that Yes is required for FGF signalling in the zebrafish embryo [63].

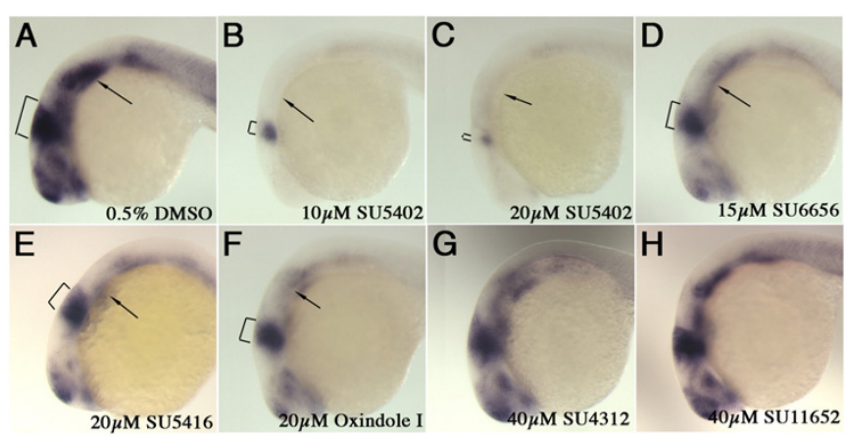

Figure 6

Expression of dusp6 in chemically treated embryos. (A-H) Lateral views of 24 hpf embryos treated with compounds indicated on bottom left and probed for the presence of dusp6 transcripts. (A) DMSO control, dusp6 is strongly expressed in the MHB (brackets), pharyngeal endoderm (arrow). (B) SU5402 at $10 \mu \mathrm{M}$ greatly suppresses dusp6 transcription, while at a higher dose, $20 \mu \mathrm{M}$ (C) dusp6 expression is almost eliminated. (D-F) SU6656, SU54I6 and Oxindole I treated embryos exhibited weaker dusp6 expression. (G \& H) SU43 I 2 and SUI I 652 did not significantly alter dusp6 expression.

The results from these experiments support the use of transgenic FGF reporter lines to screen for small molecules that affect FGF signalling. Given the rapid nature of these screens as d2EGFP expression can be suppressed in just 6 hours, it is likely that a chemical screen would identify compounds that directly modulate FGF signalling.

\section{Suppression of dusp6 expression in embryos treated with FGF inhibitors}

To confirm that suppression of d2EGFP fluorescence reflects reduced FGF target gene transcription, we analyzed dusp6 mRNA expression in wildtype embryos treated with chemical inhibitors (Figure 6). As predicted, compounds that suppressed fluorescence in $\mathrm{Tg}$ (Dusp6:d2EGFP) $)^{p t 6}$ embryos also resulted in a reduction of dusp6 transcripts (Figure 6B to 6F). SU5402, SU6656, SU5416 and Oxindole I all suppressed dusp6 expression after $10 \mathrm{hr}$ treatment at the dose indicated. In particular, dusp6 expression within the dorsal diencephalon, pharyngeal endoderm and optic stalks were greatly reduced in embryos treated with SU5402, S6656, SU5416 and Oxindole I (Figure 6B-6F). However, dusp6 expression in the MHB was either completely eliminated by SU5402 or only mildly affected by the other compounds in treated embryos (Figure 6B-6F). These results suggest that the MHB represents a region of highest levels of FGF activity in the embryo during these developmental stages. Finally, compounds that did not alter overall d2EGFP fluorescence (SU11652 and SU4312) in treated FGF reporter line also did not exhibit changes in $d u s p 6$ transcript levels (Figure 6G \&6H). 

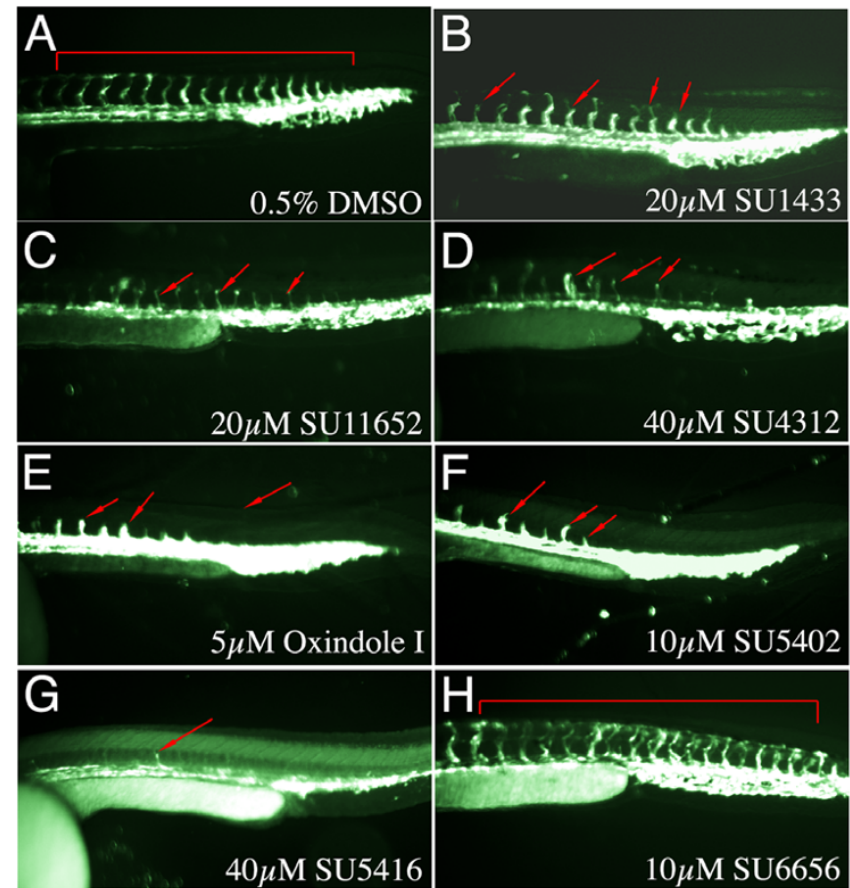

Figure 7

Specificity of Indolinones towards FGFR versus VEGFR signalling. (A-H) Lateral trunk views of $32 \mathrm{hpf}$ $\mathrm{Tg}$ (Flil:EGFP)yl embryos treated with the chemicals shown on the bottom left. (A) DMSO control shows embryo with normal expression of EGFP within the intersegmental vessels.

(B) $20 \mu \mathrm{M} \mathrm{SUI} 433$, (C) SUI I652, (D) SU43 I 2 (E) Oxindole I, (F) SU5402, and (G) SU54I6 all suppressed ISV outgrowth as indicated by red arrows. (H) SU6656 in contrast did not alter ISV formation.

Specificity of small molecule FGF signalling inhibitors can be evaluated with Tg(Fli:EGFP)yl transgenic embryos

Since the results obtained with the VEGFR inhibitors, SU11652, SU1433 and SU4312, were predominantly negative and did not alter d2EGFP expression in the FGF reporter embryos, we wanted to confirm that these compounds were permeable in the embryo and have an effect on zebrafish VEGR signalling. One approach is to test the activity of these compounds in a zebrafish angiogenesis assay. Such assays have been developed to screen for novel antiangiogenic compounds that can block the formation of the zebrafish intersegmental vessels (ISV), and transgenic lines that delineate the ISV have been used for such assays $[35,64]$. For our studies we analysed ISV outgrowth in the $\mathrm{Tg}$ (Fli1:EGFP) ${ }^{\gamma 1}$ line that expresses eGFP under the control of the Fli1 promoter [65]. To determine the effects of these VEGFR inhibitors on ISV outgrowth, we treated $\mathrm{Tg}$ (Fli1:EGFP) ${ }^{\gamma 1}$ transgenic embryos at 24 hpf for 8 hours. Treatment within this limited temporal window would reveal whether a compound could specifically block VEGFR signalling and ISV outgrowth as somites, dorsal aorta and posterior cardinal vein would have already been established at $24 \mathrm{hpf}$. Furthermore previous studies have highlighted the requirement for active VEGFR signalling that is relayed through PLC $\gamma$ and AKT to form the ISV using a similar time frame [64-66].

We first treated $\mathrm{Tg}$ (Fli1:EGFP) ${ }^{\gamma 1}$ embryos with SU1433, SU11652 and SU4312, compounds that did not alter fluorescence in the FGF reporter embryos, to determine if these compounds can block VEGFR signalling in the zebrafish. As predicted, all three compounds suppressed ISV outgrowth in the transgenic embryos, thus exhibiting specificity towards VEGFRs, and confirming that these compounds are permeable in the embryo (Figure $7 \mathrm{~B}-\mathrm{D}$ ). Similar effects on ISV outgrowth were noted with Oxindole I, a compound that blocked FGF signalling, however at a much lower dose $(5 \mu \mathrm{M})$ that was required to block fluorescence in $\mathrm{Tg}$ (Dusp6:d2EGFP) ${ }^{p t 6}$ embryos (Figure 7E). This result suggests that Oxindole I has a higher activity towards VEGFR signalling. We next assayed the effects of the FGF receptor inhibitor, SU5402, on ISV outgrowth. SU5402 treated $\mathrm{Tg}$ (Fli1:EGFP) ${ }^{\gamma 1}$ embryos also resulted in the suppression of ISV formation (Figure 7F). The original description of SU5402 had provided evidence that this compound would interact with the ATP binding domain

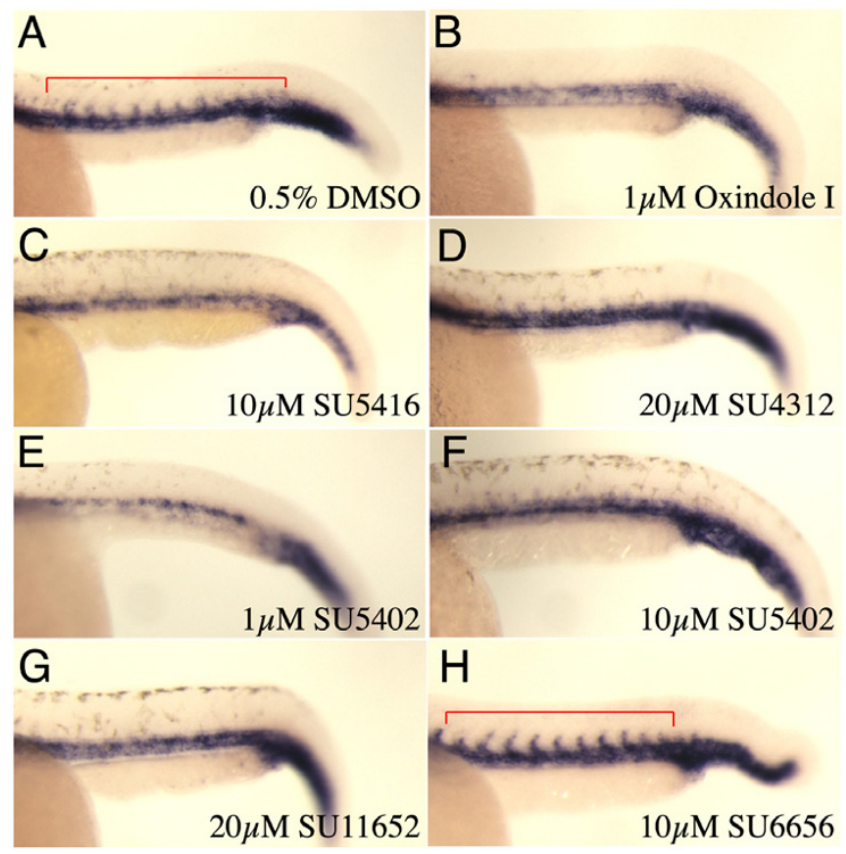

Figure 8

Expression of flil in intersegmental vessels in indolinone treated embryos. (A-H) Lateral trunk views of fli mRNA expression within the ISV. (A) DMSO control shows vessel sprouts at 28 hpf. (B-G) Embryos treated with the compounds indicated show loss of ISV sprouts, while in (H) SU6656 did not affect these vessels. 
of the VEGFR and was supported by unpublished data that SU5402 did block VEGFR signalling in tissue culture experiments, yet SU5402 is often used as a specific FGFR inhibitor. Thus we stress that the effects of SU5402 treatment may not exclusively reflect a blockade of FGFRs.

SU4312 and SU5416, two compounds that have previously been shown to inhibit VEGF signalling also inhibited ISV outgrowth (Figure 7F \&7G) [51,54,67]. Thus SU5416 strongly suppressed both VEGF and FGF signalling in our assays, while SU4312 was specific for VEGF signalling. In contrast, treatment of $T g(F l i: E G F P)^{\gamma 1}$ embryos with the inhibitor of Src kinases, SU6656, did not block ISV outgrowth, suggesting that VEGF signalling does not require Src kinases to direct vessel formation (Figure $7 \mathrm{H}$ ).

To confirm our findings that VEGFR inhibitors did indeed block intersegmental vessel outgrowth, we performed in situ hybridisation studies to detect the presence of these vessels through fli1 expression. Treatment of embryos with $1 \mu \mathrm{M}$ Oxindole $1,10 \mu \mathrm{M}$ SU5416, $20 \mu \mathrm{M}$ SU4312, 1 $\mu \mathrm{M}$ SU5402, or with $20 \mu \mathrm{M}$ SU11652 prevented ISV formation as determined by loss of fli1 expression in the intersegmental sprouts (Figure 8B-G). Again these results confirm that SU5402 can block VEGFR signalling in the zebrafish and is not a specific inhibitor of FGFRs. In contrast, expression of fli1 within the ISV was unaffected in embryos treated with $10 \mu \mathrm{M}$ SU6656, confirming the notion that Src kinases are not required in ISV outgrowth (Figure 8H).

\section{Discussion}

In this paper we present the generation of a zebrafish transgenic line that reports on FGF signalling during embryonic development. Expression of d2EGFP in these embryos is regulated by FGF signalling and maps to domains where activated ERKs have been detected [7-12]. Time-lapse imaging of transgenic embryos shows the dynamic expression of d2EGFP throughout embryogenesis. These movies demarcate the formation of Kupffer's vesicle, as d2EGFP labels the DFCs from gastrulation onwards. Since Kupffer's vesicle plays a critical role in the determination of left-right polarity these lines will provide a valuable tool to access the integrity of Kupffer's vesicle formation in left-right polarity mutants $[41,42]$. Furthermore, these lines provides a way to identify DFCs in post-gastrulation but pre-Kupffer's vesicle stages in live embryos. To our knowledge this is the only line that specifically labels the DFCs with fluorescence as they migrate during gastrulation to eventually form Kupffer's vesicle. This is in contrast to the Tg(twhh:GFP) line that also expresses GFP in Kupffer's vesicle, as GFP expression was only detected once the vesicle had formed at the 6-somite stage [68].
While a majority of the d2EGFP expression resembles that of dusp 6 mRNA during development, a notable exception is the lack of d2EGFP expression within the developing somites and tail bud, two domains where FGFs and Erk activation have been described $[3,12,31]$. Since only 10 $\mathrm{Kb}$ of upstream promoter sequence was used in generating the reporter construct, it is likely that the somitic and tail bud enhancers elements are not part of this sequence.

The expression of d2EGFP is under the control of FGF signalling as manipulation of FGF activity in transgenic embryos resulted in altered d2EGFP expression. These lines will provide useful tools to analyze the integrity of FGF signalling under experimental conditions such as antisense MOs injections or in mutant strains. Previously, Balciunas et al. described a transposon-mediated enhancer trapped line that contains an EGFP reporter inserted $30 \mathrm{~Kb}$ within the Dusp6 locus (ET7 line) [69]. In the ET7 transgenic embryos, EGFP is detected within the $\mathrm{MHB}$, pharyngeal endoderm and somites at $24 \mathrm{hpf}$, suggesting that Dusp6 enhancers may regulate the expression of eGFP [69]. However, it has not been demonstrated if EGFP expression in the ET7 embryos can respond to experimental changes in FGF signalling [69]. We believe that $T g$ (Dusp6:d2EGFP) ${ }^{p t 6}$ represent the first description of vertebrate transgenic embryos that can report on FGF activity in vivo.

We have used these transgenic lines to validate an approach to screen for small molecules that can modulate FGF signalling. Our results show that within 6 hours of treatment with the FGFR inhibitor, SU5402, complete suppression of de2GFP expression ensued, confirming that these lines can rapidly identify compounds that modulate FGF signal transduction in the embryo. Since FGFRs are members of a larger family of receptor tyrosine kinases we wanted to determine if compounds that are structurally related to SU5402 could also block FGF signalling in the embryo. We determined the specificity of several VEGFR and PDGFR inhibitors that contain the same indolinone backbone as SU5402. From our studies it was clear that SU5416 and Oxindole I, two inhibitors of VEGFR could block d2EGFP expression in the FGF reporter embryos. Furthermore, from these studies we also revealed a putative role for Src kinases in relaying FGF signals in the zebrafish as treatment with Src kinase inhibitors, PP2 and SU6656, suppressed d2EGFP fluorescence. Since several members of this kinase family are expressed early and within cells that receive FGF signals in the developing embryo, including Yes and Fyn, the preferred targets of SU6656, it is reasonable to think that these Kinases are involved in FGF signalling [70-72].

We have coupled the pilot screen with the $T g$ (Fli1:EGFP) ${ }^{y 1}$ transgenic line to assay the specificity of these compounds 
between FGFR and VEGFR signalling. We confirm that SU5416, SU4312, SU11652, Oxindole I and surprisingly SU5402 exhibited inhibitory effects on ISV outgrowth and probably a result of VEGFR inhibition. In the original study, a crystal structure of SU5402 complexed with the FGFR1 kinase domain highlighted the exact peptide residues that interfaced with the compound [48]. Since these residues are also highly conserved with the VEGFRs kinase domain, it was postulated that SU5402 could interact with the VEGFRs [48]. Furthermore the authors refer to unpublished observations that SU5402 did inhibit VEGFR signalling in living cells [48]. Our studies reveal that SU5402 can block ISV outgrowth, which we interpret as a result of VEGFR inhibition in the zebrafish embryo. Alternatively, our data could imply that FGFRs are required for ISV outgrowths given that FGFs are known to play a role in angiogenesis [53]. However from the published literature, FGFR1-4 are not expressed in the ISV to support the role for this pathway in vasculogenesis $[25,26]$. SU5402 has been used extensively as a FGFR inhibitor in many studies. In light of these findings, other experiments should be considered to test whether SU5402 might also block VEGFR signalling to elicit the observed phenotypes.

\section{Conclusion}

This study describes the generation of transgenic

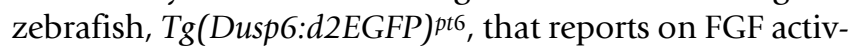
ity during development. The expression of d2EGFP mirrors the expression of several FGF ligands in the early embryo and will provide a tool to analyse FGF signalling under various experimental conditions. We have performed a pilot screen to validate these lines in chemical screens to identify novel compounds that can modulate FGF activity. This rapid screening protocol can be coupled with the $\mathrm{Tg}$ (Fli1:EGFP) ${ }^{\gamma 1}$ line to eliminate compounds that can potentially cross react with the VEGFR pathway. Finally, acridine orange staining will further eliminate toxic compounds, thus by following these procedures it is possible to identify chemicals that specifically modulate FGF signalling in vivo.

\section{Methods \\ Generation of $\operatorname{Tg}$ (Dusp6:d2EGFP) zebrafish}

A Dusp6 BAC clone was identified by PCR from BAC DNA pools as directed by manufacturers protocols (Genome Systems). A $10 \mathrm{~Kb}$ Kpn 1 fragment was identified that contain parts of Exon 1 (441 bp) and approximately $9.5 \mathrm{~Kb}$ of upstream promoter sequence (see Figure 1A). This Kpn1 fragment was subcloned into the Kpn1 site of pSce1d2EGFP vector ( $\mathrm{pSce} 1$ vector with d2EGFP cDNA cloned into the multiple cloning site). 20 pg of pDusp6:d2EGFP plasmid DNA was injected into the 1-cell embryo with I-Sce 1 (New England Biolabs, Ipswich, MA) restriction enzyme as described in [37]. These Founder F0 injected embryos were raised to adulthood and incrossed to identify transgenic founders. We identified 10 founder lines that expressed d2EGFP within regions of known FGF activity in the developing embryo. Four lines exhibited strong expression throughout development and were maintained. $T g$ (Dusp6:d2EGFP) ${ }^{p t 6}$ was used predominantly in this study.

\section{Zebrafish Microinjection of RNA and antisense Morpholinos}

$10 \mathrm{pg} f g f 8 m R N A, 20 \mathrm{ng}$ control-MO(5'-CCTCTTACCTCAGTTACAATTTATA-3'), and 10 ng $f \& f 8-M O$ (5'GAGTCTCATGTTTATAGCCTCAGTA-3') was injected into the 2-cell stage transgenic embryos as described by Tsang et. al and Araki et. al, respectively [13,73]. Morpholinos were obtained from Gene-tools inc. (Philomath, OR). Embryos were incubated for the desired stage before visualisation under a Leica stereomicroscope and photographed by a Retiga Exi camera (Qimaging, Burnaby, BC Canada). Images were analyzed in Photoshop CS (Adobe, San Jose, CA).

\section{In situ hybridisation}

Zebrafish embryos were fixed in $4 \%$ paraformaldehyde and whole mount in situ hybridisation was preformed with d2EGFP, dusp6 and fli1 RNA probes [11]. In Situ methodology as described in Kudoh et. al. [14].

\section{Chemical treatment of transgenic embryos}

Five $T g$ (Dusp6:d2EGFP) ${ }^{p t 6}$ embryos at $24 \mathrm{hpf}$ were arrayed into individual wells in a 96-well plate. $100 \mu \mathrm{l}$ of E3, $0.5 \%$ DMSO solution was added along with compound at the dose indicated. SU5402 was kindly provided by Pfizer. SU1498, SU11652, AG1433 (SU1433), SU6656, Oxindole I, were all obtained from Calbiochem (EMD biosciences, Inc. San Diego, CA) and SU5416, SU4312 from Sigma-Aldrich (St. Louis, MO). Embryos were analyzed at 6-8 hour post treatment after manual dechorinonation and treatment with tricaine to immobilise for photography. Treated embryos were photographed under the same settings for exposure, gain and magnification for each picture using a MZFLIII (leica) microscrope and fluorescent illumination for GFP using endow cube (Chroma Technology Corp., Rockingham, VT). Qimaging software and the Retiga Exi camera (Qimaging, Burnaby, BC Canada) was used to capture the images. Each experiment was repeated three times to show reproducibility of the assay and at least 4 of the 5 treated embryos exhibited the same phenotype. For treatment of $T g$ (Fli:EGFP) ${ }^{y 1}$ embryos, five transgenic embryos were placed into 96-well plates at 24 hpf and incubated with compound until $32 \mathrm{hpf}$. Treated embryos were manually dechorionated and photographed as described for the FGF reporter assay. Images were analyzed in Adobe Photoshop CS (San Jose, CA) and false coloured under the same parameters. 


\section{Zebrafish imaging}

Tg(Dusp6:d2EGFP) ${ }^{p t 6}$ embryos were placed into a MatTek glass bottom culture dish (MatTek Corp.) at gastrula stage ( $6 \mathrm{hpf}$ ) and held in place with $1 \%$ low melting point agarose. Embryos were photographed under low magnification differential contract (DIC) microscopy and fluorescent illumination for GFP using endow cube (Chroma Technology Corp., Rockingham, VT) at $5 \mathrm{~min}$ intervals until $24 \mathrm{hpf}$. Images were analyzed and processed into movies with Metamorph imaging software (Molecular Devices, Dowlingtown PA).

\section{Authors' contributions}

The experiments described in this paper were planned, conducted and analyzed by GAM, SCW and MT as a joint effort. MT isolated the Dusp6 promoter, generated the transgenic lines, described the expression of d2EGFP, and performed experiments to determine that these lines are responsive to FGFs. MT and GAM conceived the chemical screen test and GAM performed the experiments detailed in the pilot chemical screen and the in situ hybridisation. SCW was responsible for the time-lapse imaging of these lines. MT drafted the manuscript and all authors read and approved the final version.

\section{Additional material}

\section{Additional file 1}

Movie showing d2EGFP expression in Dorsal Forerunner Cells (DFCs). Movie of developing $\operatorname{Tg}$ (Dusp6:d2EGFP) ${ }^{\mathrm{pt} 6}$ embryo from late gastrula stage until bud stage. The movie shows a group of fluorescent cells, the DFCs as they coalesce towards the caudal region of the embryo.

Click here for file

[http://www.biomedcentral.com/content/supplementary/1471213X-7-62-S1.mov]

\section{Additional file 2}

Movie showing d2EGFP expression in the developing embryo. Time-laspe imaging of developing $\mathrm{Tg}$ (Dusp6: $\mathrm{d} 2 \mathrm{EGFP}) \mathrm{p}^{\mathrm{t} 6}$ embryo from gastrula stage until 24-somite stage. Movie highlights the dynamic expression of d2EGFP within the developing embryo. The movie of the fluorescent embryo was overlayed onto the DIC-imaged movie to show clearly the developing embryo. Note expression of d2EGFP within the developing hindbrain, MHB and Kupffer's vesicle.

Click here for file

[http://www.biomedcentral.com/content/supplementary/1471213X-7-62-S2.mov]

\section{Additional file 3}

Movie showing d2EGFP expression in Kupffer's vesicle. Time-laspe imaging of $\mathrm{Tg}$ (Dusp6:d2EGFP) ${ }^{\mathrm{pt} 6}$ embryo from gastrula stage until 24-somite stage. GFP movie showing the formation of Kupffer's vesicle from the 6somite stage right until after this structure collapses at the 20-somite stage. Note Kupffer's vesicle cells collapse inwards and begin to migrate towards the tail bud.

Click here for file

[http://www.biomedcentral.com/content/supplementary/1471213X-7-62-S3.mov]

\section{Additional file 4}

Movie showing d2EGFP expression in Kupffer's vesicle with DIC overlay. This is the same movie as Supplemental Movie 3, but overlayed with the DIC-imaged movie to show structures of the developing embryo.

Click here for file

[http://www.biomedcentral.com/content/supplementary/1471-

213X-7-62-S4.mov]

\section{Additional file 5}

Diagram of the small molecules used in this study. The majority of the chemicals used in the pilot screen are related in structure and contain the indolinone backbone as described for SU5402.

Click here for file

[http://www.biomedcentral.com/content/supplementary/1471213X-7-62-S5.png]

\section{Acknowledgements}

We would like to thanks Drs Tom Smithgall, Michael Rebagliati, Neil Hukriede, Beth Roman and Igor Dawid for insightful discussion, and critical reading of the manuscript. SU5402 was kindly provided by Pfizer. The I-SceI meganuclease vector and the $\mathrm{Tg}(\mathrm{Fll} I$ :EGFP)yl line was provided by $\mathrm{Dr}$ J. Wittbrodt and Dr Brant Weinstein, respectively. This work is supported by grants from the American Heart Association (0565400U) and Pennsylvania Department of Health to MT, and from the National Institutes of Health (IU54 RR0224I-0I) to SCW.

\section{References}

I. Rhinn M, Brand M: The midbrain--hindbrain boundary organizer. Curr Opin Neurobiol 200I, I I ( I):34-42.

2. Martin GR: The roles of FGFs in the early development of vertebrate limbs. Genes Dev 1998, I 2(II): I57I-1586.

3. Furthauer M, Thisse C, Thisse B: A role for FGF-8 in the dorsoventral patterning of the zebrafish gastrula. Development 1997, 124(2I):4253-4264.

4. Thisse B, Thisse $C$ : Functions and regulations of fibroblast growth factor signaling during embryonic development. Dev Biol 2005, 287(2):390-402.

5. Powers CJ, McLeskey SW, Wellstein A: Fibroblast growth factors, their receptors and signaling. Endocr Relat Cancer 2000, 7(3): 165-197.

6. Tsang M, Dawid IB: Promotion and attenuation of FGF signaling through the Ras-MAPK pathway. SCi STKE 2004, 2004(228):pel7.

7. Corson LB, Yamanaka Y, Lai KM, Rossant J: Spatial and temporal patterns of ERK signaling during mouse embryogenesis. Development 2003, I 30(19):4527-4537.

8. Schohl A, Fagotto F: Beta-catenin, MAPK and Smad signaling during early Xenopus development. Development 2002, I 29(I):37-52.

9. Sawada A, Shinya M, Jiang YJ, Kawakami A, Kuroiwa A, Takeda H: Fgfl MAPK signalling is a crucial positional cue in somite boundary formation. Development 200I, I 28(23):4873-4880.

10. Shinya M, Koshida S, Sawada A, Kuroiwa A, Takeda H: Fgf signalling through MAPK cascade is required for development of the subpallial telencephalon in zebrafish embryos. Development 200I, 128(2I):4I53-4164.

II. Tsang M, Maegawa S, Kiang A, Habas R, Weinberg E, Dawid IB: A role for MKP3 in axial patterning of the zebrafish embryo. Development 2004, I 3 I ( I 2):2769-2779.

12. Lunn JS, Fishwick KJ, Halley PA, Storey KG: A spatial and temporal map of FGF/Erk I/2 activity and response repertoires in the early chick embryo. Dev Biol 2007, 302:536-552.

13. Tsang M, Friesel R, Kudoh T, Dawid IB: Identification of Sef, a novel modulator of FGF signalling. Nat Cell Biol 2002, 4(2): $165-169$.

14. Kudoh T, Tsang M, Hukriede NA, Chen X, Dedekian M, Clarke CI, Kiang A, Schultz S, Epstein JA, Toyama R, Dawid IB: A gene expression screen in zebrafish embryogenesis. Genome Res 200I, II(12): 1979-1987. 
15. Furthauer M, Lin W, Ang SL, Thisse B, Thisse C: Sef is a feedbackinduced antagonist of Ras/MAPK-mediated FGF signalling. Nat Cell Biol 2002, 4(2): I70-I74

16. Eblaghie MC, Lunn JS, Dickinson RJ, Munsterberg AE, Sanz-Ezquerro JJ Farrell ER, Mathers J, Keyse SM, Storey K, Tickle C: Negative feedback regulation of FGF signaling levels by PystI/MKP3 in chick embryos. Curr Biol 2003, I3(12): 1009-1018.

17. Kawakami Y, Rodriguez-Leon J, Koth CM, Buscher D, Itoh T, Raya A $\mathrm{Ng}$ JK, Esteban CR, Takahashi S, Henrique D, Schwarz MF, Asahara H, Izpisua Belmonte JC: MKP3 mediates the cellular response to FGF8 signalling in the vertebrate limb. Nat Cell Biol 2003 5(6):5|3-5|9.

18. Echevarria D, Martinez S, Marques S, Lucas-Teixeira V, Belo JA: Mkp3 is a negative feedback modulator of Fgf8 signaling in the mammalian isthmic organizer. Dev Biol 2005, 277(I): I | 4- I 28

19. Li C, Scott DA, Hatch E, Tian X, Mansour SL: Dusp6 (Mkp3) is a negative feedback regulator of FGF-stimulated ERK signaling during mouse development. Development 2007, I34(I): I67-176.

20. Smith TG, Karlsson M, Lunn JS, Eblaghie MC, Keenan ID, Farrell ER Tickle C, Storey KG, Keyse SM: Negative feedback predominates over cross-regulation to control ERK MAPK activity in response to FGF signalling in embryos. FEBS Lett 2006 , 580( I 7):4242-4245.

21. Vieira C, Martinez S: Experimental study of MAP kinase phosphatase-3 (Mkp3) expression in the chick neural tube in relation to Fgf8 activity. Brain Res Brain Res Rev 2005, 49(2): I58-I66.

22. Smith TG, Sweetman D, Patterson M, Keyse SM, Munsterberg A Feedback interactions between MKP3 and ERK MAP kinase control scleraxis expression and the specification of rib progenitors in the developing chick somite. Development 2005 , 132(6): $|305-13| 4$

23. David NB, Saint-Etienne L, Tsang M, Schilling TF, Rosa FM: Requirement for endoderm and FGF3 in ventral head skeleton formation. Development 2002, I 29( I 9):4457-4468.

24. Furthauer M, Reifers F, Brand M, Thisse B, Thisse C: sprouty4 acts in vivo as a feedback-induced antagonist of FGF signaling in zebrafish. Development 200I, I 28( I 2):2 I 75-2 I86.

25. Scholpp S, Groth C, Lohs C, Lardelli M, Brand M: Zebrafish fgfrl is a member of the fgf 8 synexpression group and is required for fgf8 signalling at the midbrain-hindbrain boundary. Dev Genes Evol 2004, 2 I 4(6):285-295.

26. Tonou-Fujimori $N$, Takahashi $M$, Onodera $H$, Kikuta $H$, Koshida $S$, Takeda H, Yamasu K: Expression of the FGF receptor 2 gene (fgfr2) during embryogenesis in the zebrafish Danio rerio. Gene Expr Patterns 2002, 2(3-4): | 83-188.

27. Raible F, Brand M: Tight transcriptional control of the ETS domain factors Erm and Pea3 by Fgf signaling during early zebrafish development. Mech Dev 2001, 107 (1-2): 105-117.

28. Roehl H, Nusslein-Volhard $C$ : Zebrafish pea3 and erm are general targets of FGF8 signaling. Curr Biol 200 I, I I (7):503-507.

29. Munchberg SR, Ober EA, Steinbeisser H: Expression of the Ets transcription factors erm and pea3 in early zebrafish development. Mech Dev 1999, 88(2):233-236.

30. Reifers F, Adams J, Mason IJ, Schulte-Merker S, Brand M: Overlappin and distinct functions provided by fgf $\mathrm{I} 7$, a new zebrafish member of the Fgf8/I7/I8 subgroup of Fgfs. Mech Dev 2000 99( I-2):39-49.

31. Reifers F, Bohli H, Walsh EC, Crossley PH, Stainier DY, Brand M: Fgf8 is mutated in zebrafish acerebellar (ace) mutants and is required for maintenance of midbrain-hindbrain boundary development and somitogenesis. Development 1998 , I 25(13):2381-2395.

32. MacRae CA, Peterson RT: Zebrafish-based small molecule discovery. Chem Biol 2003, I 0( I 0):901-908.

33. Peterson RT, Link BA, Dowling JE, Schreiber SL: Small molecule developmental screens reveal the logic and timing of vertebrate development. Proc Natl Acad Sci U S A 2000 97(24): 12965-12969.

34. Zon LI, Peterson RT: In vivo drug discovery in the zebrafish. Nat Rev Drug Discov 2005, 4(I):35-44.

35. Cross LM, Cook MA, Lin S, Chen JN, Rubinstein AL: Rapid analysis of angiogenesis drugs in a live fluorescent zebrafish assay. Arterioscler Thromb Vasc Biol 2003, 23(5):911-912

36. Li X, Zhao X, Fang Y, Jiang X, Duong T, Fan C, Huang CC, Kain SR: Generation of destabilized green fluorescent protein as a transcription reporter. I Biol Chem 1998, 273(52):34970-34975.

37. Thermes V, Grabher C, Ristoratore F, Bourrat F, Choulika A, Wittbrodt J, Joly JS: I-Scel meganuclease mediates highly efficient transgenesis in fish. Mech Dev 2002, I I 8(I-2):9|-98.

38. Cooper MS, D'Amico LA: A cluster of noninvoluting endocytic cells at the margin of the zebrafish blastoderm marks the site of embryonic shield formation. Dev Biol 1996, I80(I): 184-I98.
39. Melby AE, Warga RM, Kimmel CB: Specification of cell fates at the dorsal margin of the zebrafish gastrula. Development 1996 I 22(7):2225-2237.

40. Hashimoto H, Rebagliati M, Ahmad N, Muraoka O, Kurokawa T, Hibi $M$, Suzuki T: The Cerberus/Dan-family protein Charon is a negative regulator of Nodal signaling during left-right patterning in zebrafish. Development 2004, I 31 (8): I74 I-I753.

4I. Essner J], Amack JD, Nyholm MK, Harris EB, Yost HJ: Kupffer's vesicle is a ciliated organ of asymmetry in the zebrafish embryo that initiates left-right development of the brain, heart and gut. Development 2005, I 32(6): I 247-I 260.

42. Kramer-Zucker AG, Olale F, Haycraft CJ, Yoder BK, Schier AF, Drummond IA: Cilia-driven fluid flow in the zebrafish pronephros, brain and Kupffer's vesicle is required for normal organogenesis. Development 2005, I32(8): | 907-1921.

43. Long S, Ahmad N, Rebagliati M: The zebrafish nodal-related gene southpaw is required for visceral and diencephalic left-right asymmetry. Development 2003, I30(I I):2303-2316

44. Albertson RC, Yelick PC: Roles for fgf8 signaling in left-right patterning of the visceral organs and craniofacial skeleton. Dev Biol 2005, 283(2):310-32I.

45. Halpern ME, Liang JO, Gamse JT: Leaning to the left: laterality in the zebrafish forebrain. Trends Neurosci 2003, 26(6):308-3 I3.

46. Concha ML, Burdine RD, Russell C, Schier AF, Wilson SW: A noda signaling pathway regulates the laterality of neuroanatomical asymmetries in the zebrafish forebrain. Neuron 2000 , 28(2):399-409.

47. Lun K, Brand M: A series of no isthmus (noi) alleles of the zebrafish pax2.I gene reveals multiple signaling events in development of the midbrain-hindbrain boundary. Development 1998, I 25( I 6):3049-3062.

48. Mohammadi M, McMahon G, Sun L, Tang C, Hirth P, Yeh BK, Hubbard $S R$, Schlessinger J: Structures of the tyrosine kinase domain of fibroblast growth factor receptor in complex with inhibitors. Science 1997, 276(53 | 4):955-960.

49. Delaune E, Lemaire P, Kodjabachian L: Neural induction in Xenopus requires early FGF signalling in addition to $B M P$ inhibition. Development 2005, I32(2):299-310.

50. Maroon H, Walshe J, Mahmood R, Kiefer P, Dickson C, Mason I: Fgf3 and $\mathrm{Fgf8}$ are required together for formation of the otic placode and vesicle. Development 2002, I 29(9):2099-2108.

5I. Fong TA, Shawver LK, Sun L, Tang C, App H, Powell TJ, Kim YH, Schreck $R$, Wang $X$, Risau W, Ullrich A Hirth KP, McMahon G: SU54I6 is a potent and selective inhibitor of the vascular endothelial growth factor receptor (FIk-I/KDR) that inhibits tyrosine kinase catalysis, tumor vascularization, and growth of multiple tumor types. Cancer Res 1999, 59(I):99-106.

52. Giles FJ, Cooper MA, Silverman L, Karp JE, Lancet JE, Zangari M, Shami PJ, Khan KD, Hannah AL, Cherrington JM, Thomas DA, Garcia-Manero G, Albitar M, Kantarjian HM, Stopeck AT: Phase II study of SU54 I 6--a small-molecule, vascular endothelial growth factor tyrosine-kinase receptor inhibitor--in patients with refractory myeloproliferative diseases. Cancer 2003 97(8): 1920-1928.

53. Cross MJ, Claesson-Welsh L: FGF and VEGF function in angiogenesis: signalling pathways, biological responses and therapeutic inhibition. Trends Pharmacol Sci 200I, 22(4):20I-207.

54. Kendall RL, Rutledge RZ, Mao X, Tebben AJ, Hungate RW, Thomas KA: Vascular endothelial growth factor receptor KDR tyrosine kinase activity is increased by autophosphorylation of two activation loop tyrosine residues. I Biol Chem 1999, 274( ( 0):6453-6460.

55. Liao AT, Chien MB, Shenoy N, Mendel DB, McMahon G, Cherrington JM, London CA: Inhibition of constitutively active forms of mutant kit by multitargeted indolinone tyrosine kinase inhibitors. Blood 2002, I 00(2):585-593.

56. Heryanto B, Lipson KE, Rogers PA: Effect of angiogenesis inhibitors on oestrogen-mediated endometrial endothelial cell proliferation in the ovariectomized mouse. Reproduction 2003, I 25(3):337-346.

57. Strawn LM, McMahon G, App H, Schreck R, Kuchler WR, Longhi MP Hui TH, Tang C, Levitzki A, Gazit A, Chen I, Keri G, Orfi L, Risau W, Flamme I, Ullrich A, Hirth KP, Shawver LK: Flk-I as a target for tumor growth inhibition. Cancer Res 1996, 56( I 5):3540-3545.

58. Boguslawski G, McGlynn PW, Harvey KA, Kovala AT: SUI498, an inhibitor of vascular endothelial growth factor receptor 2 causes accumulation of phosphorylated ERK kinases and inhibits their activity in vivo and in vitro. J Biol Chem 2004, 279(7):5716-5724

59. Arbiser JL Larsson $\mathrm{H}$, Claesson-Welsh L, Bai X, LaMontagne K, Weiss SW, Soker S, Flynn E, Brown LF: Overexpression of VEGF I 2 I in immortalized endothelial cells causes conversion to slowly 
growing angiosarcoma and high level expression of the VEGF receptors VEGFR-I and VEGFR-2 in vivo. Am J Pathol 2000, I 56(4): | 469- | 476.

60. Boilly B, Vercoutter-Edouart AS, Hondermarck H, Nurcombe V, Le Bourhis $X$ : FGF signals for cell proliferation and migration through different pathways. Cytokine Growth Factor Rev 2000, I I (4):295-302.

6I. Kusakabe M, Masuyama N, Hanafusa $H$, Nishida E: Xenopus FRS2 is involved in early embryogenesis in cooperation with the Src family kinase Laloo. EMBO Rep 200I, 2(8):727-735.

62. Tatton L, Morley GM, Chopra R, Khwaja A: The Src-selective kinase inhibitor PPI also inhibits Kit and Bcr-Abl tyrosine kinases. J Biol Chem 2003, 278(7):4847-4853.

63. Blake RA, Broome MA, Liu X, Wu J, Gishizky M, Sun L, Courtneidge SA: SU6656, a selective src family kinase inhibitor, used to probe growth factor signaling. Mol Cell Biol 2000, 20(23):9018-9027.

64. Chan J, Bayliss PE, Wood JM, Roberts TM: Dissection of angiogenic signaling in zebrafish using a chemical genetic approach. Cancer Cell 2002, I (3):257-267.

65. Lawson ND, Weinstein BM: In vivo imaging of embryonic vascular development using transgenic zebrafish. Dev Biol 2002, 248(2):307-3।8.

66. Lawson ND, Mugford JW, Diamond BA, Weinstein BM: phospholipase $C$ gamma-I is required downstream of vascular endothelial growth factor during arterial development. Genes Dev 2003, I 7(I I): |346-|35|.

67. Serbedzija GN, Flynn E, Willett CE: Zebrafish angiogenesis: a new model for drug screening. Angiogenesis 1999, 3(4):353-359.

68. Du SJ, Dienhart M: Zebrafish tiggy-winkle hedgehog promoter directs notochord and floor plate green fluorescence protein expression in transgenic zebrafish embryos. Dev Dyn 200I, 222(4):655-666.

69. Balciunas D, Davidson AE, Sivasubbu S, Hermanson SB, Welle Z, Ekker SC: Enhancer trapping in zebrafish using the Sleeping Beauty transposon. BMC Genomics 2004, 5(I):62.

70. Sharma D, Holets L, Zhang X, Kinsey WH: Role of Fyn kinase in signaling associated with epiboly during zebrafish development. Dev Biol 2005, 285(2):462-476.

7I. Jopling C, den Hertog J: Fyn/Yes and non-canonical Wnt signalling converge on RhoA in vertebrate gastrulation cell movements. EMBO Rep 2005, 6(5):426-43I.

72. Tsai WB, Zhang X, Sharma D, Wu W, Kinsey WH: Role of Yes kinase during early zebrafish development. Dev Biol 2005, 277(I): $129-14 \mid$.

73. Araki I, Brand M: Morpholino-induced knockdown of fgf8 efficiently phenocopies the acerebellar (ace) phenotype. Genesis 200I, 30(3): I57-I59.
Publish with Biomed Central and every scientist can read your work free of charge

"BioMed Central will be the most significant development for disseminating the results of biomedical research in our lifetime. "

Sir Paul Nurse, Cancer Research UK

Your research papers will be:

- available free of charge to the entire biomedical community

- peer reviewed and published immediately upon acceptance

- cited in PubMed and archived on PubMed Central

- yours - you keep the copyright
BioMedcentral 